\title{
ON APPROXIMATION TO AN ARBITRARY FUNCTION OF A COMPLEX VARIABLE BY POLYNOMIALS*
}

\author{
BY \\ J. L. WALSH
}

1. Introduction. In the theory of functions of a complex variable there are many problems relating to uniform approximation (or what is essentially the same thing, to uniform expansion) of an arbitrary function by means of polynomials, which have not yet been completely solved. Let us mention a few examples.

When can a function $f(z)$ defined on a closed point set $M$ be uniformly approximated on $M$ by polynomials in $z$ ? Of what point sets $M$ is it true that every function continuous on $M$ can be approximated in this way? Of what closed point sets $M$ is it true that every function $f(z)$ continuous on $M$ and analytic at every interior point of $M$ can be uniformly approximated? What conformal maps can be approximately performed by means of polynomials, with an error uniformly and arbitrarily small?

The present paper does not give a complete answer to any of these questions, but gives new results connected with each of them, particularly when the point sets $M$ in question consist of Jordan curves or arcs which do not necessarily refrain from intersecting. Hitherto only such configurations as consist of non-intersecting curves have been treated, with reference to uniform approximation of functions. The results of the present paper are the most general results which can be obtained in answer to the questions raised, concerning point sets $M$ which are composed of a finite number of Jordan arcs, curves, and regions.

If a point set $M$ extends to infinity and is closed, the only function $f(z)$ continuous on $M$ which can be uniformly approximated on $M$ by polynomials is a constant. Hence throughout this paper we consider, unless the contrary is explicitly stated, only point sets which do not extend to infinity.

The results which are to be obtained here are proved by the use of modern work in conformal mapping-a theory which has yielded most of the recent results on approximation of functions of a complex variable.

2. Statement of some results obtained; connection with the literature. Let us indicate more specifically the advance made in the present paper. Runge's theorem is classical:

\footnotetext{
* Presented to the Society, December 28, 1927; received by the editors in November, 1927.
} 
If the function $f(z)$ is analytic in a closed Jordan region, ${ }^{*}$ then in that closed region $f(z)$ can be uniformly expanded in a series of polynomials in $z$.

The following results have more recently been established: $\dagger$

Theorem I. If the function $f(z)$ is analytic interior to a Jordan curve and continuous in the corresponding closed region, then in that closed region $f(z)$ can be uniformly expanded in a series of polynomials in $z$.

ThEOREM II. If the function $f(z)$ is continuous on a Jordan arc, then on that arc $f(z)$ can be uniformly expanded in a series of polynomials in $z$.

The following more general theorems, among others, are to be proved here:

Theorem III. If the two Jordan cirves $C_{1}$ and $C_{2}$ are exterior to each other except for a single common point $z=\alpha$, and if the functions $f_{1}(z)$ and $f_{2}(z)$ are respectively analytic interior to $C_{1}$ and $C_{2}$ and continuous in the corresponding closed regions, and if $f_{1}(\alpha)=f_{2}(\alpha)$, then there exists a series of polynomials in $z$ which converges uniformly to the sum $f_{1}(z)$ in the closed interior of $C_{1}$ and uniformly to the sum $f_{2}(z)$ in the closed interior of $C_{2}$.

Theorem IV. If the bounded point set $M$ consists of a finite number of Jordan arcs and does not divide the plane, then an arbitrary function $f(z)$ continuous on $M$ is developable on $M$ in a uniformly convergent series of polynomials in $z$.

3. A theorem on the conformal mapping of variable regions. For later use in the study of expansions we require the following theorem:

Theorem V. In the z-plane let the Jordan curves $C_{1}$ and $C_{2}$ lie exterior to each other, except that they have the single point $A(z=\alpha)$ in common. Let $B_{1}$ and $B_{2}$ be fixed points of $C_{1}$ and $C_{2}$ respectively. Let the Jordan curve $K_{n}$, $n=1,2, \cdots$, contain the interiors of $C_{1}$ and $C_{2}$ and consist (at least for $n$ sufficiently great) of an arc of $C_{1}$ which contains $B_{1}$, an arc of $C_{2}$ which contains $B_{2}$, and of two arcs of the circle with center $A$ and radius $1 / n$. If $z^{\prime}=\omega_{n}(z)$ denotes the function that maps the interior of $K_{n}$ onto the interior of $C_{1}$ so

* We emphasize the fact that the region here is not the most general simply connected region of the plane; proof that the theorem breaks down in this more general case will be found on pp. 432433 of the paper referred to in the next following footnote. Misunderstanding of this fact caused the statement of an erroneous theorem in the Encyklopädie, II C4, Bieberbach, Neuere Untersuchungen uber Funktionen von komplexen Variablen, p. 49.

† By application, suggested by Caratheodory, of a theorem on conformal mapping due to Courant. See Walsh, Mathematische Annalen, vol. 96 (1926), pp. 430-436; 437-450. 
that a fixed point $D$ interior to $C_{1}$ and a fixed direction at that point remain invariant, then we have

$$
\lim _{n \rightarrow \infty} \omega_{n}(z)=\omega(z)= \begin{cases}z, & z \text { on or interior to } C_{1}, \\ \alpha, & z \text { on or interior to } C_{2},\end{cases}
$$

uniformly on the closed point set consisting of the curves $C_{1}$ and $C_{2}$ and their interiors.

We do not prove (1) directly, but find it more convenient to map the interiors of $C_{1}$ and of $K_{n}$ onto the interior of the unit circle $\Gamma$ in the $\tau$-plane, in such a way that $D$ is transformed into the origin and the given direction at $D$ into the same direction at $\tau=0$. Denote by $\tau=\phi(z), \phi_{n}(z)$ the respective mapping functions. Then (1) is entirely equivalent to the uniform approach to a limit:

$$
\lim _{n \rightarrow \infty} \phi_{n}(z)= \begin{cases}\phi(z), & z \text { on or interior to } C_{1}, \\ \phi(\alpha), & z \text { on or interior to } C_{2} .\end{cases}
$$

For the two functions $z^{\prime}=\omega_{n}(z)$ and $\tau=\phi_{n}(z)$ are obtained from each other by the transformation $\tau=\phi\left(z^{\prime}\right)$ and its inverse, both of which are continuous transformations in the closed region consisting of $C_{1}$ and its interior. We proceed, then, to the proof of (2).

The result

$$
\lim _{n \rightarrow \infty} \phi_{n}(z)=\phi(z), z \text { on or interior to } C_{1}^{\prime},
$$

uniformly, when $C_{1}^{\prime}$ is a fixed Jordan curve interior to $C_{1}$, follows from a theorem due to Bieberbach.* But by results of Lindelöf, points interior

* Göttinger Nachrichten, 1913, pp. 552-560. The names of Caratheodory (Mathematische Annalen, vol. 73 (1912), pp. 107-144) and of Courant (Hurwitz-Courant, Funktionentheorie, Berlin, 1925, pp. 385-387) should also be mentioned here.

We apply the theorem of Bieberbach, 广oc. cit., p. 557, although he considers the mapping of regions $K_{n}$ on variable regions, and with the auxiliary conditions (in our notation) $\phi_{n}(0)=0, \phi_{n}{ }^{\prime}(0)=1$, where $D$ is the point $z=0$. This involves no real difficulty, for we need merely modify our $\phi_{n}(z)$ and $\phi(z)$ by proper multiplicative constants to bring about this change; the new functions map the interior of $K_{n}$ on variable circles whose Kern (Carathéodory) is the transform of the interior of $C_{1}$. Uniform convergence of these modified functions $\phi_{n}(z)$ results from Bieberbach's theorem, and implies the uniform convergence of our original functions $\phi_{n}(z)$ as used in the present paper.

The connection of this entire subject, the conformal mapping of variable regions, with Lebesgue's work on harmonic functions (Rendiconti del Circolo Matematico di Palermo, vol. 24 (1907), pp. 371-402) seems not to have been pointed out in the literature, but we could in fact use his results instead of those of Bieberbach. We outline briefly the method. By the results of Lebesgue (pp. 398399), the Green's function (as defined by Osgood, Funktionentheorie, Leipzig, 1912, p. 630) for the interior of $K_{n}$ approaches uniformly in the closed interior of $C_{1}$ (strictly speaking, the single point $D$ is an exception) the Green's function for the interior of $C_{1}$. The sequence of functions conjugate to the Green's functions for the interior of $K_{n}$ approaches likewise the function conjugate to the Green's function for the interior of $C_{1}$ and uniformly in any closed region interior to $C_{1}$, when this conjugate function is defined properly for our auxiliary conditions $\phi_{n}{ }^{\prime}(0)>0, \phi^{\prime}(0)>0$. It follows that the mapping function $\phi_{n}(z)$ converges uniformly to the limit $\phi(z)$ in any closed region interior to $C_{1}$. 
to $C_{1}$ and $K_{n}$ and within a distance $\delta$ of the boundary are transformed by $\tau=\phi(z), \phi_{n}(z)$ into points within a distance $\eta(\delta)$ of the boundary of $\Gamma$, where $\eta(\delta)$ is independent of $n$ and approaches zero with $\delta .^{*}$ Moreover, a point $P$ interior to $C_{1}$ or $K_{n}$ which can be connected to a point $Q$ of the boundary of $C_{1}$ or $K_{n}$ by a Jordan arc (interior to $C_{1}$ or $K_{n}$ ) of diameter less than $\delta$ is transformed into points $P^{\prime}, P_{n}$ interior to $\Gamma$ whose distances from the points $Q^{\prime}, Q_{n}$ corresponding to $Q$ are respectively less than $\theta(\delta)$, where $\theta(\delta)$ is independent of $n$ and approaches zero with $\delta$.

Let an arbitrary positive $\epsilon$ be given. Choose $\delta$ so that $\theta(\delta)<\epsilon / 6$. Choose the curve $C_{1}^{\prime}$ so that every point $R$ of $C_{1}^{\prime}$ is within a distance $\delta / 2$ of $C_{1}$ and is interior to $C_{1}$, and so that every point $S$ on or within $C_{1}$ but not within $C_{1}^{\prime}$ can be joined interior to $C_{1}$ by a Jordan arc of diameter less than $\delta / 2$ to some point of $C_{1}^{\prime}$. Choose also $N>4 / \delta$. Then an arbitrary point $S$ on or within $C_{1}$ but not within $C_{1}^{\prime}$, even such a point $S$ in the neighborhood of $A$, can be joined interior to $C_{1}$ by a Jordan arc of diameter less than $\delta$ to some point $Q$ of $C_{1}$ and of $K_{n}$ for $n>N$.

Denote by $Q^{\prime}, R^{\prime}, S^{\prime}$, and $Q_{n}, R_{n}, S_{n}$ the transforms of $Q, R, S$ respectively under the transformations $\tau=\phi(z), \tau=\phi_{n}(z)$. Then if the points $R$ and $S$ previously considered can be joined within $C_{1}$ by a Jordan arc of diameter less than $\delta / 2$, the points $R$ and $S$ can be joined within $C_{1}$ by a Jordan arc of diameter less than $\delta$ to some point $Q$ (the same point $Q$ for both $R$ and $S$ ) of $C_{1}$ and of $K_{n}$, so we have

$$
R^{\prime} Q^{\prime}<\frac{\epsilon}{6}, \quad Q^{\prime} S^{\prime}<\frac{\epsilon}{6}, \quad R_{n} Q_{n}<\frac{\epsilon}{6}, \quad Q_{n} S_{n}<\frac{\epsilon}{6},
$$

and hence we have

$$
R^{\prime} S^{\prime}<\frac{\epsilon}{3}, \quad R_{m} S_{n}<\frac{\epsilon}{3},
$$

uniformly for all $R, S$, and $n>N$.

But by choosing $n$ sufficiently large we have by Bieberbach's result, $R^{\prime} R_{n}<\epsilon / 3$ uniformly for all $R$ on $C_{1}^{\prime}$ and for all sufficiently large $n$. If $S$ is an arbitrary point on or within $C_{1}$ but not within $C_{1}^{\prime}$, there exists some point $R$ of $C_{1}^{\prime}$ which can be joined within $C_{1}$ to $S$ by a Jordan arc of diameter less than $\delta / 2$. Thus we have, by combining the inequalities already derived,

$$
S^{\prime} S_{n}=\left|\phi(z)-\phi_{n}(z)\right|<\epsilon
$$

uniformly for all sufficiently large $n$ and for all points $S$ on or within $C_{1}$ but not within $C_{1}^{\prime}$. This, together with the result of Bieberbach,

* Lindelöf, Acta Societatis Scientiarum Fennicae, vol. 46 (1915), No. 4, Sur un principe général de l'analyse, pp. 1-35; especially pp. 15-18. 


$$
\lim _{n \rightarrow \infty} \phi_{n}(z)=\phi(z) \text {, uniformly for all } z \text { on or within } C_{1}^{\prime},
$$

yields immediately the first part of (2),

$$
\lim _{n \rightarrow \infty} \phi_{n}(z)=\phi(z) \text {, uniformly for all } z \text { on or within } C_{1} \text {. }
$$

If each point of the curve $C_{1}^{\prime}$ is within a distance $\delta / 2$ of the curve $C_{1}$, the transform $J_{1}^{\prime}$ of $C_{1}^{\prime}$ is everywhere within a distance $\eta(\delta / 2+1 / n)$ of the circle $\Gamma$, and the transform $J_{2}^{\prime}$ of $C_{2}$ is a Jordan curve lying outside of $J_{1}^{\prime}$, under the transformation $\tau=\phi_{n}(z)$. Every point of $J_{2}^{\prime}$ not a point of $\Gamma$ lies exterior to $J_{1}^{\prime}$, and within some circle of radius $\theta(2 / n)$, for all points of $C_{2}$ not points of $K_{n}$ can be connected by a Jordan curve interior to $K_{n}$ whose diameter is less than $2 / n$ to a single point $Q$ of $K_{n}$. We have already proved, however,

$$
\lim _{n \rightarrow \infty} \phi_{n}(\alpha)=\phi(\alpha)
$$

from which follows, since $\phi_{n}(\alpha)$ lies on $J_{2}^{\prime}$, the second part of (2), and (2) is completely established.

We remark that Theorem $\mathrm{V}$ can be extended to include the case of several (even an infinity of suitably restricted) Jordan curves $C_{2}$, each having but a single point in common with $C_{1}$; the proof already given holds without essential change. In the theorem as stated it is not essential that the curves $K_{n}$ should be composed partly of arcs of circles. It is sufficient (although even this is not necessary for the proof) that the arcs of $K_{n}$ not part of $C_{1}$ or $C_{2}$ lie in a circle whose center is $A$ and whose radius approaches zero as $n$ becomes infinite.

The writer has had the privilege of discussing the proof of Theorem $\mathbf{V}$ with Dr. Karl Löwner of Berlin, and hereby expresses his indebtedness to him for aid in formulating that proof.

4. Approximation on two point sets having a single common point. As a matter of general notation, if we denote a Jordan curve by $C_{i}$, the closed point set which consists of the curve and its interior will be denoted by $\bar{C}_{\boldsymbol{i}}$.

Our most important tool in later work is a consequence of Theorem V:

Theorem VI. Let the Jordan curves $C_{1}$ and $C_{2}$ lie exterior to each other except for a single point $A, z=\alpha$, which they have in common. Let the sequence of polynomials $\left\{P_{n}^{\prime}(z)\right\}$ converge to a function $F_{1}(z)$ defined throughout $\bar{C}_{1}$ or more generally on any point set which contains $A$ and is contained in $\bar{C}_{1}$. Similarly let the sequence of polynomials $\left\{P_{n}^{\prime \prime}(z)\right\}$ converge to a function $F_{2}(z)$ defined throughout $\bar{C}_{2}$ or more generally on any point set which contains $A$ and is contained in $\bar{C}_{2}$. Then if $F_{1}(\alpha)=F_{2}(\alpha)$, there exists a sequence of polynomials $\left\{P_{n}(z)\right\}$ such that 


$$
\begin{aligned}
& \lim _{n \rightarrow \infty}\left[P_{n}(z)-P_{n}^{\prime}(z)\right]=0, \text { uniformly for } z \text { in } \bar{C}_{1}, \\
& \lim _{n \rightarrow \infty}\left[P_{n}(z)-P_{n}^{\prime \prime}(z)\right]=0, \text { uniformly for } z \text { in } \bar{C}_{2} .
\end{aligned}
$$

It follows that in $\bar{C}_{1}$ the points of convergence of the sequences $\left\{P_{n}(z)\right\}$ and $\left\{P_{n}^{\prime}(z)\right\}$ are the same, likewise point sets of uniform convergence; in case the former sequence converges with a certain sum, the latter sequence converges with the same sum, and conversely. A similar remark applies to the sequences $\left\{P_{n}(z)\right\}$ and $\left\{P_{n}^{\prime \prime}(z)\right\}$ in $\bar{C}_{2}$.

There can easily be proved by the classical method of Runge* a result similar to Theorem VI for the case that $\bar{C}_{1}$ and $\bar{C}_{2}$ have no point in common. Thus, if in this case the sequences of polynomials $\left\{P_{n}^{\prime}(z)\right\}$ and $\left\{P_{n}^{\prime \prime}(z)\right\}$ converge in $\bar{C}_{1}$ and $\bar{C}_{2}$ respectively or on point sets contained in those regions, there exists a sequence of polynomials $\left\{P_{n}(z)\right\}$ satisfying the requirements (3). This result can also be proved easily by direct application of Theorem VI. $\dagger$ We shall apply this result later, in the proof of Theorem VII.

Theorem $\mathrm{V}$ is to be applied in the proof of Theorem VI. The function $\omega_{n}(z)$ is analytic interior to $K_{n}$, and continuous in the region $\bar{K}_{n}$, which includes of course the point set $\bar{C}_{1}+\bar{C}_{2}$. Hence by Theorem I the function $\omega_{n}(z)$ is in $\bar{K}_{n}$ uniformly developable in series of polynomials, and therefore the limit of this function, $\omega(z)$, approached uniformly, is in $\bar{C}_{1}+\bar{C}_{2}$ likewise uniformly developable in a series of polynomials.

Let us suppose for the sake of simplicity that $F_{1}(\alpha)=F_{2}(\alpha)=0$; this assumption involves no loss of generality. Choose an arbitrary sequence $\left\{\epsilon_{n}\right\}$ of positive numbers whose limit is zero. Since $\omega(z)$ is uniformly developable on $\bar{C}_{1}+\bar{C}_{2}$ in a series of polynomials, there exists a polynomial $Q_{n}^{\prime}(z)$ such that

$$
\begin{aligned}
& \left|Q_{n}^{\prime}(z)-P_{n}^{\prime}(z)\right|<\frac{\epsilon_{n}}{2}, \quad z \text { on } \bar{C}_{1}, \\
& \left|Q_{n}^{\prime}(z)-P_{n}^{\prime}(\alpha)\right|<\frac{\epsilon_{n}}{2}, \quad z \text { on } \bar{C}_{2} .
\end{aligned}
$$

* See for example Montel, Séries des polynomes d une variable complexe, Paris, 1910, Ch. IV.

$\dagger$ We sketch this suggested proof. Choose a point $A, z=\alpha$, not in $\bar{C}_{1}$ or $\bar{C}_{2}$; apply Theorem VI to the polynomial sequences $\left\{(z-\alpha) P_{n}^{\prime}(z)\right\}$ and $\left\{(z-\alpha) P_{n}^{\prime \prime}(z)\right\}$ considered in new Jordan regions $\bar{C}_{1}{ }^{\prime}$ and $\bar{C}_{2}{ }^{\prime}$, which have the single common point $A$ and which contain $\bar{C}_{1}$ and $\bar{C}_{2}$ respectively. We thus arrive at a sequence of polynomials which we write $\left\{P_{n}^{\prime \prime}(z)\right\}$ and consider in $\bar{C}_{1}$ and $\bar{C}_{2}$. But in $\bar{C}_{1}$ and $\bar{C}_{2}$ the function $1 /(z-\alpha)$ can be expanded uniformly in a series of polynomials, and hence the sequence $\left\{P_{n}^{\prime \prime}(z) /(z-\alpha)\right\}$ may be replaced by a sequence of polynomials $\left\{P_{n}(z)\right\}$ having in $\bar{C}_{1}$ and $\bar{C}_{2}$ the properties required. 
The polynomial $Q_{n}^{\prime}(z)$ may be taken equal to $P_{n}^{\prime}[\Omega(z)]$, where $\Omega(z)$ is a polynomial which approximates $\omega(z)$ sufficiently closely on $\bar{C}_{1}+\bar{C}_{2}$.

Similarly there exists a polynomial $Q_{n}^{\prime \prime}(z)$ such that

$$
\begin{aligned}
& \left|Q_{n}^{\prime \prime}(z)-P_{n}^{\prime \prime}(z)\right|<\frac{\epsilon_{n}}{2}, \quad z \text { on } \bar{C}_{2}, \\
& \left|Q_{n}^{\prime \prime}(z)-P_{n}^{\prime \prime}(\alpha)\right|<\frac{\epsilon_{n}}{2}, \quad z \text { on } \bar{C}_{1} .
\end{aligned}
$$

Then the polynomial $Q_{n}(z)=Q_{n}^{\prime}(z)+Q_{n}^{\prime \prime}(z)$ satisfies the inequalities

$$
\begin{aligned}
& \left|Q_{n}(z)-P_{n}^{\prime \prime}(\alpha)-P_{n}^{\prime}(z)\right|<\epsilon_{n}, z \text { on } \bar{C}_{1}, \\
& \left|Q_{n}(z)-P_{n}^{\prime}(\alpha)-P_{n}^{\prime \prime}(z)\right|<\epsilon_{n}, z \text { on } \bar{C}_{2},
\end{aligned}
$$

so that by virtue of

$$
\lim _{n \rightarrow \infty} P_{n}^{\prime}(\alpha)=F_{1}(\alpha)=0, \quad \lim _{n \rightarrow \infty} P_{n}^{\prime \prime}(\alpha)=F_{2}(\alpha)=0,
$$

the sequence $\left\{Q_{n}(z)\right\}$ satisfies the requirements of Theorem VI.

5. Approximation on $m$ given point sets. Theorem III follows immediately from Theorems I and VI. A generalization of Theorem VI which considers not merely two but an arbitrary number of Jordan curves will turn out to be convenient:

THEOREM VII. Let the point set $M$ be composed of the closed interiors of the Jordan curves $C_{1}, C_{2}, \cdots, C_{m}$; we suppose that these curves lie exterior to each other, except that $C_{i}$ and $C_{i}(i, j=1,2, \cdots, m, i \neq j)$ may have a single point $z=\alpha_{i j}$ (but no more) in common. We suppose also that the system of curves $C_{i}$ divides the plane into precisely $m+1$ distinct regions. Then if the sequence of polynomials $\left\{P_{n}^{(i)}(z)\right\}, i=1,2, \cdots, m$, converges to a limit function $F_{i}(z)$ defined on the entire point set $\bar{C}_{i}$ or a part of $i t$, and if finally $F_{i}\left(\alpha_{i j}\right)=F_{j}\left(\alpha_{i j}\right)$ for all points $\alpha_{i j}$ which exist, ${ }^{*}$ then there exists a sequence of polynomials $\left\{P_{n}(z)\right\}$ such that

$$
\lim _{n \rightarrow \infty}\left[P_{n}(z)-P_{n}^{(i)}(z)\right]=0 \text {, uniformly on } \bar{C}_{i}, i=1,2, \cdots, m .
$$

From (4) follows, as in Theorem VI, that in $\bar{C}_{i}$ the points of convergence and limits of the sequences $\left\{P_{n}(z)\right\}$ and $\left\{P_{n}^{(i)}(z)\right\}$ are the same, likewise point sets of uniform convergence, and so on.

* Here there are two interpretations possible, (a) that when $\alpha_{i j}$ exists, both functions $F_{i}(8)$ and $F_{j}(z)$ are required to be defined and equal there, or (b) that when $\alpha_{i j}$ exists and when $F_{i}\left(\alpha_{i j}\right)$ and $F_{i}\left(\alpha_{i j}\right)$ are both defined, then these two functional values shall be equal. We understand here the former interpretation. 
Theorem VII has already been established for the case $m=2$. Let us proceed by induction, assuming the theorem true for $m=\mu-1$ and proving the theorem for $m=\mu$. At least one of the curves $C_{i}$, let us say for definiteness $C_{1}$, has not more than one point in common with the totality of other curves. Otherwise every curve $C_{i}$ would possess at least two such points, there would exist a new (i.e., distinct from the $C_{i}$ ) Jordan curve consisting only of arcs of the curves $C_{i}$, and the $\mu$ curves $C_{i}$ would separate the plane into more than $\mu+1$ regions. Hence there exists a Jordan curve $K$ which is exterior to $C_{1}$, except that $K$ may have a single point in common with $C_{1}$, and such that $\bar{C}_{i}$ belongs to $\bar{K}$ for $i=2,3, \cdots, m=\mu$. Theorem VII now follows, on the one hand by application of Theorem VII for $m=\mu-1$ to the point set $\bar{C}_{2}+\bar{C}_{3}+\cdots+\bar{C}_{\mu}$, and on the other hand by application to $C_{1}$ and $K$ either of Theorem VI or of the result (method of Runge) similar to Theorem VI, according as $C_{1}$ and $K$ have or have not a common point.

The following theorem, of which Theorem III is a special case, results from Theorem VII without further argument:

ThEOREM VIII. If the point set $M$ satisfies the conditions of Theorem VII and if the function* $f(z)$ is analytic interior to $C_{i}$ and continuous on $M$, then on $M$ the function $f(z)$ can be developed in a uniformly convergent series of polynomials.

6. A general result on approximation. Our most general theorem will now be proved; both Theorems VIII and IV are included here as special cases.

TheOREM IX. Let the point set $M$ consist of the closed interiors of the Jordan curves $C_{1}, C_{2}, \cdots, C_{m}$, and of the Jordan arcs $B_{1}, B_{2}, \cdots, B_{n}$. Suppose the curves $C_{i}(i \neq j)$ and the arcs $B_{1}, B_{2}, \cdots, B_{n}$ are all exterior to the curve $C_{i}$ except that possibly a single point (but no more) of $C_{i}$ or $B_{k}$ may coincide with a point of $C_{j}$. Suppose finally that the curves $C_{1}, \cdots, C_{m}, B_{1}, \cdots$, $B_{n}$ do not separate the plane into more than $m+1$ distinct regions. Then an arbitrary function analytic in the interior points of $M$ and continuous on $M$ can be developed uniformly on $M$ in series of polynomials.

We suppose the notation such-and this involves no loss of generalitythat no point of an $\operatorname{arc} B_{i}$ other than an end point is a point of $C_{k}$ or of $B_{i}(i \neq j)$.

There can be drawn Jordan curves $C_{m+1}, C_{m+2}, \cdots, C_{m+n}$ so that $B_{i}$ belongs to $\bar{C}_{m+i}$, so that no two of the curves $C_{i}(i=1,2, \cdots, m+n)$

* Of course, not necessarily a monogenic analytic function. 
have more than a single point in common, so that these curves lie exterior to each other except for these common points, and so that the curves $C_{1}$ $\ldots, C_{m+n}$ do not divide the plane into more than $m+n+1$ distinct regions. This construction is possible by enclosing the arc $B_{i}$ in a Jordan curve $C_{m+i}$, drawn sufficiently close to $B_{i}$ and having on it no point of $M$ other than the end points of $B_{i}$, and having no point in common with other $\operatorname{arcs} B_{i}$ or with a curve $C_{k}(k=1,2, \cdots, m+n, k \neq m+i)$ except such points as $B_{i}$ has in common with those curves. Theorem IX then follows immediately, by application of Theorems I, II, and VII.

The kind of function $f(z)$ considered in Theorem IX is the most general function which can be uniformly expanded on $M$ in a series of polynomials. For the sum of such a uniformly convergent series is continuous on $M$ and analytic in the interior points of $M$.

Theorem IX breaks down if we omit from the hypothesis the requirement that the curves $C_{1}, \cdots, C_{m}, B_{1}, \cdots, B_{n}$ shall not separate the plane into more than $m+1$ distinct regions. Proof of this fact is essentially to be found in the discussion to be given later, in $\S 8$.

7. Remarks on conformal mapping. Theorem IX has interesting applications to conformal mapping. If we have in the $z$-plane the point set $M$ of that theorem and in the w-plane a second point set $M^{\prime}$ consisting of Jordan curves $C_{1}^{\prime}, C_{2}^{\prime}, \cdots, C_{m}^{\prime}$, and of Jordan arcs $B_{1}^{\prime}, B_{2}^{\prime}, \cdots, B_{n}^{\prime}$, having the other properties mentioned in the theorem, and finally if the point sets $M$ and $M^{\prime}$ can be mapped on each other one-to-one and continuously without exception and conformally so far as concerns the interiors of $C_{\boldsymbol{i}}$ and $C_{\boldsymbol{i}}^{\prime}$, then this mapping can be accomplished with any desired degree of approximation by means of polynomials, from the $z$-plane to the $w$-plane and the reverse. For the mapping functions satisfy all the requirements of Theorem IX.

Two arbitrary point sets $M$ and $M^{\prime}$ having the properties mentioned in the theorem and which are topologically equivalent cannot always be mapped on each other in this way. For example, a circle $C_{1}$ with four Jordan $\operatorname{arcs} B_{i}$ abutting on it cannot be so mapped on an arbitrary circle $C_{1}^{\prime}$ with four Jordan arcs abutting on it. For the four points common to $C_{1}$ and $B_{i}$ cannot in general be transformed into the four points common to $C_{1}^{\prime}$ and $B_{i}^{\prime}$; the most general conformal map of a circle onto another circle involves three real parameters. But in every case if $M$ and $M^{\prime}$ satisfy the requirements of Theorem IX, if they are topologically equivalent, and if $C_{i}$ has no more than three points in common with $C_{1}+C_{2}+\cdots+C_{i-1}+C_{i+1}$ $+\cdots+C_{m}+B_{1}+\cdots+B_{n}$, then $M$ and $M^{\prime}$ can be mapped on each other continuously and one-to-one, conformally so far as concerns the in- 
teriors of $C_{i}$ and $C_{i}^{\prime}$, and this transformation can be accomplished with an arbitrary degree of approximation by means of a polynomial in $z$ or $w$.

To the point set $M$ of Theorem IX there may be adjoined without altering the truth of the theorem an arbitrary number of additional points $P$, provided these new points have at most a finite number of limit points and that the entire point set, $M$ plus the new points, is closed. The property still holds, that if $f(z)$ is analytic in the interior points of $M$ and continuous on the entire point set, then on that entire point set $f(z)$ can be uniformly expanded in a series of polynomials. The details here are easy and are omitted.* This remark has obvious applications to conformal mapping by means of polynomials.

8. Approximation by rational functions. We return to the questions raised in $\$ 1$. The following theorem is extremely easy to prove, but perhaps so near to these questions that it is worth stating:

Let $M$ be a limited, closed point set. Then a necessary and sufficient condition that every function $f(z)$ analytic on $M$ be uniformly developable on $M$ in a series of polynomials, is that $M$ be the complementary set (with respect to the entire plane) of an infinite region.

Here the function $f(z)$ is not required to be a monogenic analytic function, but is merely required to be (regular-) analytic at every point of $M$.

This condition on $M$ is sufficient, for there can be drawn-for instance, by the help of a network of squares-a finite number of Jordan curves all exterior to each other such that each curve has on and within it only points of analyticity of $f(z)$, and such that every point of $M$ is interior to one of these curves. The fact that $f(z)$ is developable follows by Runge's results.

The condition on $M$ is necessary. For let us assume the contrary, that there exists a point $P$ not a point of $M$ but which cannot be joined to the point at infinity by a curve which does not meet $M$. Then all points $Q$ which can be joined to $P$ by curves which do not meet $M$ form a region $R^{\prime}$ all of whose boundary points are points of $M$. A region $R$ exists which is simply-connected, which contains all points of $R^{\prime}$, and whose boundary points are all points of $M$. Then the function $f(z)=1 /(z-p)$, where $P$ is the point

* Compare a remark due to Szegö and the present writer, Walsh, loc. cit., p. 443, footnote.

Many other points sets $N$, in particular certain of them containing an infinity of Jordan arcs $B$, can be made essentially to come under this theorem. For addition of new points to $N$ may enable us to construct thereby a point set $M$ satisfying the requirements of Theorem IX. A function continuous on $N$ is easily extended in definition so as to be continuous also on $M$. Of course we add in the general case, where an arbitrary function $f(z)$ is concerned, no interior points to $N$, nor do we increase the number of Jordan curves of $N$. 
$z=p$, is analytic in all points of $M$ but cannot be developed on $M$ uniformly in a series of polynomials.*

The subject of approximation of arbitrary functions by means of rational functions instead of by polynomials is an interesting one which has received little attention in the literature. All the questions raised in the first part of the present paper can properly be raised for approximation by means of rational functions instead of by means of polynomials, and these questions are still, in their ultimate generality, unanswered. One extremely simple theorem is perhaps worth mentioning, although the proof presents no difficulty. This theorem and the next one stated hold even for points sets which extend to infinity, provided that to the plane a single point at infinity be adjoined:

Let $M$ be an arbitrary closed point set of the plane. If the function $f(z)$ is analytic in every point of $M$, then $f(z)$ can be expanded on $M$ in a uniformly convergent series of rational functions of $z$.

Deeper lying results can be established by the use of conformal mapping, and the methods of the present paper can be used here to some extent. As an illustration of results that can be obtained in this way, we state without proof at this time the following theorem:

If the point set $M$ consists of two Jordan curves $C_{1}$ and $C_{2}$ which have only a single point in common, then an arbitrary function $f(z)$ continuous on $M$ can be uniformly expanded on $M$ in a series of rational functions. If the fixed points $P_{1}, P_{2}, P_{3}$ are chosen arbitrarily, one in each of the three regions into which $M$ divides the plane, then each rational function of the series can be chosen so that it has no poles except in $P_{1}, P_{2}$, and $P_{3}$.

* See Walsh, loc. cit., p. 434.

If $M$ is a limited, closed point set, then a necessary and sufficient condition that a given function $f(z)$ analytic on $M$ be uniformly developable on $M$ in a series of polynomials, is that $f(z)$ or its analytic extension (i.e. all analytic extensions which can be obtained from points of $M$ along paths which do not elsewhere meet $M)$ be analytic in every point which cannot be connected with the point at infinity by a curve which does not meet $M$.

This condition can be otherwise expressed. All points which can be connected with the point at infinity by curves which do not meet $M$ form a region $B$. The condition is then that $f(z)$ or its analytic extension be analytic in every point of the point set complementary (with respect to the entire plane) to the region $B$.

HARVARD UNIVERSITY, Cambridge, Mass. 